\title{
Biodiesel from Microalgae: The Use of Multi-Criteria Decision Analysis for Strain Selection
}

\author{
Ezinne Nwokoagbara, Akeem K Olaleye, Meihong Wang* \\ Process and Energy Systems Engineering Group, School of Engineering, University of \\ Hull, HU6 7RX, United Kingdom \\ *Corresponding author. Tel: +44 1482 466688; Email address: Meihong.Wang@hull.ac.uk
}

\begin{abstract}
Microalgae strain selection is a vital step in the production of biodiesel from microalgae. In this study, MultiCriteria Decision Analysis (MCDA) methodologies are adopted to resolve this problem. The aim of this study is to identify the best microalgae strain for viable biodiesel production. The microalgae strains considered here are Heynigia sp., Scenedesmus sp., Niracticinium sp., Chlorella vulgaris, Chlorella sorokiniana and Auxenochlorella protothecoides. The five MCDA methods used to evaluate different strains of microalgae are Analytic Hierarchy Process (AHP), Weighted Sum Method (WSM), Weighted Product Method (WPM), Discrete Compromise Programming (DCP) and Technique for the Order of Preference to the Ideal Solution (TOPSIS). Pairwise comparison matrices are used to determine the weights of the evaluation criteria and it is observed that the most important evaluation criteria are lipid content and growth rate. From the results, Scenedesmus $s p$. is selected as the best microalgae strain among the six alternatives due to its high lipid content and relatively fast growth rate. The AHP is the most comprehensive of the five MCDA methods because it considers the importance of each criterion and inconsistencies in the rankings are verified. The implementation of the MCDA methods and the results from this study provide an idea of how MCDA can be applied in microalgae strain selection.
\end{abstract}

Keywords: $\quad$ Microalgae, Strain Selection, Biodiesel, Multi-Criteria Decision Analysis

\subsection{Introduction}

The interest in renewable energy sources such as biofuels is increasing due to unstable crude oil prices, possible dwindling of fossil fuel reserves, lingering concerns about the environment, and the need for energy security [1]. The conversion of biomass resources results in biofuels; these resources are energy 
sources that can be replenished naturally at almost the same rate as they are used. Wood, crops, waste, animal residue and organic marine life (such as algae) are various forms of biomass.

Biofuels have oxygen levels of 10 to $45 \mathrm{wt} \%$ (dry), while fossil-based fuels have essentially none, making the chemical properties of biofuels very different from those of their fossil-based counterparts [2]. This high oxygen content leads to more efficient and "cleaner" combustion. Biofuels typically have very low sulphur and nitrogen levels, thus reducing the levels of sulphur and nitrogen oxide released upon combustion [3]. $\mathrm{CO}_{2}$ neutrality is a primary advantage of biofuels [1]. This is based on the concept that during the growth phase of biomass, it consumes as much $\mathrm{CO}_{2}$ as is released when burnt as a biofuel (i.e. the same number of carbon atoms are recycled).

Microalgae is a third generation biofuel source with several advantages over terrestrial crops owing to its high potential yield of biofuels and relatively faster growth rates [4]. $\mathrm{CO}_{2}$ can be captured and used in large scale cultivation of algae for biofuel production [2]. Fig. 1 illustrates $\mathrm{CO}_{2}$ mitigation (carbon neutrality) using microalgae as an energy resource.

Microalgae can be grown on non-arable land or in large water bodies utilizing ocean or waste water; hence eliminating the competition for land and fresh water with food crops. With wastewater containing nutrients such as urea, nitrogen, phosphorus and potassium, the cultivation of microalgae can be mutually beneficial because the microalgae can utilize the nutrients for growth while the wastewater is treated by the algae [5]. The process of producing biodiesel from microalgae can be summarized in four major steps: 1) Microalgae cultivation; 2) Harvesting; 3) Algal oil extraction; 4) Transesterification to produce biodiesel.

MCDA involves making decisions in the presence of multiple, potentially conflicting criteria. The goal of MCDA is the selection of the "best" alternative from pre-specified alternatives described in terms of multiple attributes [6, 7]. The first complete exposition of MCDA was given in 1976 by Keeney and Raiffa [8].

Previous studies indicate that more than 50,000 species of microalgae exist, but only about 30,000 have been studied and analysed [5]. These strains have different physical, chemical and biological properties, and can affect the production process in different ways. These differences make microalgae strain selection an important task.

There is insufficient information in literature about the application of MCDA in microalgae strain selection for biodiesel production. This study aims to address this insufficiency by evaluating six microalgae strains C 2015, Elsevier. Licensed under the Creative Commons Attribution-NonCommercial-NoDerivatives 4.0 International 
using six MCDA methods to determine the best strain for biodiesel production. In the methodology adopted, the relative importance of the decision maker's opinions of the criteria is determined by a pairwise comparison matrix using linguistic-to-numerical characterizations developed by Thomas Saaty in 1980 [9]. Table 1(a) shows a summary of the advantages and disadvantages of the common MCDA methods.

. Table 1(a): Advantages and Disadvantages of MCDA methods

\section{Properties of microalgae for biodiesel production}

The criteria that can influence microalgae strain selection can be grouped into the technical, environmental, economic and social aspects [3]. These are presented in Table 1(b). , The most important properties of microalgae for biodiesel production are growth rate, lipid content, fatty acid profile and ease of harvesting [4].

Fig. $1 \quad \mathrm{CO}_{2}$ mitigation using algae [2]

Table 1(b): Evaluation criteria used in MCDA for microalgae strain selection [3]

\subsection{Properties of microalgae and their effects on biodiesel}

\subsubsection{Growth rate}

Microalgae can double their biomass yields in timeframes as short as 3.5 hours and the average harvesting cycle is about $1-10$ days [1]. This rapid growth potential makes microalgae a viable feedstock for commercial biodiesel production [10].

\subsubsection{Lipid content}

From various literature sources, the lipid content of microalgae biomass can range from 4.5 to $80 \%$ of its dry weight and these lipids are in the form of oils [11]. The lipid content of a microalgae strain is directly proportional to the quantity of biodiesel produced; therefore the use of high lipid - producing strains result in high yields of biodiesel. Microalgae oil contains neutral and polar lipids. Neutral lipids or Triglycerides (TAG) are the most desirable components for biodiesel production from microalgae $[4,10]$. The quantity (by dry weight) and quality of the lipids contained in a microalgae strain are very important criteria for biodiesel production. Strains capable of producing more than $50 \%$ dry weight of extractable oils are viable for industrial biodiesel production [1].

(C) 2015, Elsevier. Licensed under the Creative Commons Attribution-NonCommercial-NoDerivatives 4.0 International 


\subsubsection{Fatty acid profile}

TAGs are esters of glycerol and three fatty acids. The fatty acids contained in microalgae oil are: Free Fatty Acids (FFA), Monounsaturated Fatty Acids (MUFA), Polyunsaturated Fatty Acids (PUFA), and Saturated Fatty Acids (SUFA) [12]. Higher percentages (by composition) of SUFA and MUFA result in biodiesel with enhanced energy yields, higher oxidative stability and higher cetane number; however, these high percentages will also lead to biodiesel with poor cold flow properties. Strains with high PUFA will result in biodiesel with higher oxidation rates but good cold flow properties [12, 13, and 14]. High FFA content causes saponification during the transesterification of microalgae oil resulting in poor yields of biodiesel [1]. The fatty acid profile of a good quality biodiesel - that strikes a balance between cold flow and other properties - should have a 5:4:1 mass ratio of C16:1, C18:1 and C14:0 [14].

\subsubsection{Ease of harvesting}

Harvesting of microalgae biomass is one of the most challenging areas of biodiesel production from microalgae. The concentration of microalgae biomass relative to the volume of liquid in the culture medium is typically between 0.3 and $5 \mathrm{~g} / \mathrm{L}$, this low biomass - to - liquid ratio has to be "concentrated" to about $300-400 \mathrm{~g} / \mathrm{L}$ (by dry weight) for industrial scale operations [15]. The small cell size $(2-20 \mu \mathrm{m})$ of the microalgae cells in addition to the high water content of the culture medium, make the harvesting a costly and energy intensive process, taking up about $20-30 \%$ the total production cost [16]. Harvesting can be carried out by one or a combination of the following methods: centrifugation, filtration, flocculation, flotation, sedimentation and ultrasonic separation. The process to adopt is mostly determined by the physical properties of the microalgae strain (shape, cell size, specific gravity) and cultivation method (open ponds, bioreactors and hybrid production systems) [10,11]. Microalgae species with the fastest growth rates are often very small in size and as a result are very difficult to harvest [11]. Filtration is a commonly used method and its use depends on the size of the microalgae being harvested, it is however an expensive process to operate because of the constant replacement of filters, low recovery rates, long processing times and energy consumed for pumping [15]. Centrifugation is the fastest method for microalgae biomass harvesting, the drawback however is the excessive energy consumption which can run as high as $3000 \mathrm{kWh} / \mathrm{t}$ [15]. Flocculation is problematic and uneconomical due to the similarities in the specific gravities of the 
microalgae cells and the culture medium; industrial quantities of flocculants are very expensive [15]. Sedimentation requires large areas of land for settling ponds and tanks. The high moisture content of the biomass recovered from sedimentation requires a secondary dewatering process, which further increases the cost of production $[15,5]$. The harvesting method selected must be energy efficient and relatively inexpensive in order to contribute to the economic feasibility of the biodiesel production process.

\subsection{Microalgae strains}

The properties of microalgae-based biodiesel depend on the strain of microalgae from which it was produced. For instance Botryococcus braunii contains terpenoid hydrocarbons while Chlorella prothothecoides contains glyceryl lipids. In literature, there are several suggested approaches for screening microalgae strains for biofuels production:

(a) Viswanath et.al noted that one of the ways of achieving best selection with productivity characteristics

is by screening based on natural habitat. The important characteristics include growth rate, oil content, fatty acid profile, robustness and resistance to contamination $[4,13]$.

(b) The approach employed by Doan et al. is similar and more elaborate by comparison. The focus of the selection was towards microalgae strains for biodiesel production. Growth rate, elevated biomass, intracellular lipid content (i.e. neutral and polar lipids) and fatty acid profile of the microalgae were the characteristics considered [13].

(c) Maharajh and Harilal $[13,17]$ of the CSIRO algal team in Australia based their characterizations for identifying microalgae with high lipid content and rapid specific growth rate.

Different growth and nutrient conditions can affect the chemical composition of microalgae strains. An example is with the Chlorella protothecoides strain - when grown under autotrophic conditions, its chemical composition was mainly protein (51\%), lipids (14\%), carbohydrate (10\%), ash (7\%) and moisture (11\%); but under heterotrophic conditions, the lipid content increased to up to $55.2 \%$ and the protein content was reduced to about $10 \%$. With further improved conditions, the lipid content can be as high as $58 \mathrm{wt} \%$ [1]. Environmental conditions also have a part to play in the selection of microalgae strains. A particular strain of microalgae cultivated in a tropical climate for instance may have markedly different properties (e.g. lipid 
content, biomass quantity, growth rate) than the same strain grown in a colder climate. The lipid content of microalgae species will typically remain unchanged provided it is grown under the same conditions [18]. The properties of the microalgae strain impacts the conversion process (microalgae to biodiesel). Strains with high FFA content, require acid pre-treatment before undergoing base-catalysed transesterification [1]. This additional step in the conversion process will have significant cost and energy implications.

Micro algae is a very expensive raw material with the cost of production of microalgae biomass typically costing about $\$ 2.95-3.80 / \mathrm{kg}$ as reported by [16] and about $\$ 25,000 / \mathrm{t}$ as reported by [19]. This cost factor is a limiting factor in the development of microalgae as a potential source of energy.

\section{MCDA methodology}

MCDA is based on deriving an overall score for the alternative being analysed. A primary advantage of MCDA is the provision of a highly structured decision-making technique. Goals, inputs, alternatives, criteria and weights serve as the core components of MCDA [20]. Within a decision-making problem, criteria are used to evaluate the performance of all the alternatives. Relative importance factors (weights) of the relevant criteria are defined by the decision maker and these factors are numerical representations of the preference of the decision maker based on background information and experience. MCDA provides a numerical score, or rating, assigned to a given alternative with respect to each criterion. Typically, one alternative does not satisfy all the important criteria; alternatives that are more beneficial for instance are usually more costly, hence some compromise or trade-off is usually evident amongst the goals [8].

\subsection{Steps for MCDA}

The basic procedure applies to all MCDA techniques involving the numerical analysis of alternatives [21]. The main difference between the types of MCDA techniques is the scoring process as each process synthesizes information differently [7]. As illustrated in Fig. 2, the main stages of MCDA [22] are:

\subsubsection{Defining the problem (Goal)}

C 2015, Elsevier. Licensed under the Creative Commons Attribution-NonCommercial-NoDerivatives 4.0 International 
The aims and features of the decision-making problem, characteristics of the proposed MCDA method and background information regarding the problem are addressed [6].

\subsubsection{Determining the relevant criteria and alternatives}

The criteria are used to evaluate the alternatives. The use of too few criteria can lead to oversimplification of the decision problem and a large number of criteria can reduce the influence of any one criterion in determining the rank of an alternative. Sometimes the criteria of interest may not be determinable and hence proxies may be used [21].

\subsubsection{Identifying the inputs}

Inputs tailor the MCDA to the specific decision making situation. Inputs to an MCDA are in form of numbers or percentages based on the discretion/preference of the decision maker. Input values are obtained from background information and experience.

\subsubsection{Weighting}

Among the criteria, some are considered as more important (having more impact on the goal) than the others. Weighting involves assigning numerical measures to the criterion according to their relative importance, these numerical measures are the "Weights" [21]. Criteria weights have direct influences on MCDA results, therefore it is important to assign weights rationally to obtain accurate results.

\section{Fig. 2 MCDA steps [22]}

Three factors are normally considered in assigning weights: the subjective preference of the decision maker, the variance degree and independence of the criteria [22].

\subsubsection{Final treatment and Aggregation}

The numerical values are processed to determine a ranking of each alternative. This step is dependent on the type of MCDA process involved [21]. In some cases, a sensitivity analysis of the results can be considered an additional step. This can be done in order to determine how changes in the weights or inputs of the criteria can affect the rankings of an alternative [6].

(C) 2015, Elsevier. Licensed under the Creative Commons Attribution-NonCommercial-NoDerivatives 4.0 International 


\subsection{MCDA methods}

For a decision-making problem, one MCDA method can be selected based on available information and the nature of the problem. More than one method can also be used and the results compared. Usually the results of all MCDA methods will be similar with only minor differences in the alternative rankings [20].

MCDA methods are often grouped into two general categories: the Value-based methods and the Outranking methods $[7,20]$.

Value based methods use a rating scale where the lower values represent the least desirable qualities and the higher values represent the more desirable qualities. The range of the rating scales is arbitrary and can be selected to meet the desires of the decision maker [20]. Value based methods include: AHP, DCP, WPM, WSM, Multi-attribute Utility Theory, Weighted Average method [20].

Outranking models compare the performance of two or more alternatives in terms of each criterion, to identify the extent to which a preference for one over the other can be asserted [7]. The preferred alternative tends to be the one that has the highest performance in the largest number of criteria [20]. Outranking techniques are most appropriate when criteria metrics are not easily aggregated, measurement scales vary over wide ranges, and units are incommensurate or incomparable [7]. Outranking methods include: Multi-attribute Value Theory and PROMETHEE.

\subsubsection{AHP}

Developed by Thomas L. Saaty in the 1970's, this method involves aggregating various facets of the decision problem into a single optimization function known as the "Objective Function". The goal of the AHP is to select the alternative that results in the greatest value of the objective function. AHP uses a quantitative comparison method based on pairwise comparisons of criteria. All individual criterion must be paired against others and the results compiled in a matrix form. A numerical scale is used to compare the criteria and the AHP method moves systematically through all pairwise comparisons of criteria and alternatives [7].

\subsubsection{WSM}

The earliest and probably most widely used method often called the "Weighted Scoring" or "Simple Additive Weighting" method $[21,23]$. In this method, criteria area "weighted" according to importance or priority and 
numerical values assigned to these priorities. The alternatives are accessed based on their ability to meet a specific criterion and a weighted product value is derived. By summing the weighted product values, the alternative most closely meeting the criteria can be determined as the best. The WSM is described by the equation:

$$
\begin{gathered}
S_{i}=\sum_{j} w_{j} s_{i j} \\
\text { where: } \sum_{j=1}^{m} w_{j}=1 \quad \text { for } i=1,2,3,4, \ldots \ldots n
\end{gathered}
$$

$\mathrm{Si}=$ score of alternative $\mathrm{i}$; the alternative with the highest score is the best; $\mathrm{s}_{\mathrm{ij}}=$ score of alternative $\mathrm{i}$ using criterion $\mathrm{j} ; \mathrm{w}_{\mathrm{j}}=$ weight for criterion $\mathrm{j}$ [23]. Criteria with different units of measure have to be normalized in order to make them non-dimensional and hence comparable on the same scale. The normalizing equations are [24]:

For maximizing/positive/benefit criteria (gets better with increasing numerical value):

$$
\bar{r}_{i j}=\frac{r_{i j}}{\max r_{i j}}
$$

For minimizing/negative/cost criteria (gets worse with increasing numerical value):

$$
\bar{r}_{i j}=\frac{\min r_{i j}}{r_{i j}}
$$

Where $\max r_{i j}=$ maximum value of criterion $j$ with respect to alternative $i$; $\min r_{i j}=$ minimum value of criterion j with respect to alternative $i$.

The major drawback of this method is that it assumes linearity of preferences which may not reflect the decision maker's preferences [25]. Another disadvantage is the lack of theoretical basis for calculating weights [23], hence the weights are usually calculated by pairwise comparisons from the AHP.

\subsubsection{WPM}

In this method, the weights become exponents associated with each attribute value: positive and negative powers for benefit and cost attributes respectively [26]. The criteria do not need to be transformed into dimensionless values by normalization due to the multiplicative nature of this method [27]. The equation for the WPM is:

(C) 2015, Elsevier. Licensed under the Creative Commons Attribution-NonCommercial-NoDerivatives 4.0 International 


$$
R_{i}=\frac{V\left(A_{i}\right)}{V\left(A^{*}\right)}=\frac{\prod_{j=1}^{n} x_{i j}^{w_{j}}}{\prod_{j=1}^{n}\left(x_{j}^{*}\right)^{w_{j}}} \quad \text { for } i=1,2,3,4, \ldots \ldots . . n
$$

$V\left(A_{i}\right)$ and $V\left(A^{*}\right)$ are the multi attribute utility functions of the ith and ideal alternatives respectively. The ideal alternative is the alternative with optimal performance attributes, when every attribute is optimized independently of each other while considering the constraints of the problem. This alternative is unfeasible in reality [28]. The use of $V\left(A^{*}\right)$ puts a numerical bound to the alternative values obtained by this method [27]. $x^{*}$ is the ideal value of the jth attribute across all the alternatives; $x_{i j}$ is the ith attribute of the jth alternative; and $R_{i}$ is the score of the ith alternative. The WPM is sometimes called dimensionless analysis because its structure eliminates any units of measure. The WPM can be considered as modification of the WSM and has been proposed as a solution to overcome some of its weaknesses [21].

\subsubsection{DCP}

A rating scale of 0 to 1 is used in this method, with a value of 1 representing the best rating and a value of 0 the worst. For maximized criteria, DCP is described as:

$$
R_{i, j}=\left[\sum_{i=1}^{n} w_{i}^{p}\left[\frac{f_{i}^{*}-f_{j}(x)}{\left|f_{i}^{*}-f_{* j}\right|}\right]^{p}\right]^{\frac{1}{p}}
$$

For minimized criteria:

$$
R_{i, j}=\left[\sum_{i=1}^{n} w_{i}^{p}\left[\frac{f_{j}(x)-f_{i}^{*}}{\left|f_{i}^{*}-f_{* j}\right|}\right]^{p}\right]^{\frac{1}{p}}
$$

$\mathrm{R}_{\mathrm{ij}}$ is the score of the ith alternative; the alternative with the lowest score is the best. $f_{i}^{*}$ is the ideal value of ith criteria; $f_{j}(x)$ is the value of the ith criteria with respect to the decision variables $x$ (actual value of the criteria); $f_{* j}$ is the anti-ideal value of the ith criteria; $\mathrm{p}$ is the exponent determining the distance function, it has a value of 1 or 2 [20].

When other MCDA methods produce alternatives with equal ranks, DCP will provide more discrimination and result in a non-equal ranking [20]. 


\subsubsection{TOPSIS}

This method selects the alternative closest to the positive ideal solution (ideal alternative) and farthest from the negative ideal solution (anti-ideal alternative) as the best [23]. TOPSIS is carried out in the following steps $[23,27]$ :

Step 1: The criteria are normalized using the vector normalization equation:

$$
\bar{r}_{i j}=\frac{x_{i j}}{\sqrt{\sum_{j=1}^{n} x_{i j}^{2}}}
$$

Step 2: The weighted normalized ratings are calculated as:

$$
v_{i j}=w_{i} * r_{i j}
$$

Where $x^{*}{ }_{i j}$ is the ith criteria of the jth alternative; $w_{i}$ is the weight of the ith attribute and $v_{i j}$ is the weighted normalized rating.

Step 3: The separation measures are the distance of each alternative from the positive or negative ideal solutions. They are denoted by the n-dimensional Euclidean distance:

$$
\begin{gathered}
S_{j}^{*}=\sqrt{\sum_{i=1}^{n}\left(v_{i j}-v_{i}^{*}\right)^{2}} \\
S_{j}^{-}=\sqrt{\sum_{i=1}^{n}\left(v_{i j}-v_{i}^{-}\right)^{2}}
\end{gathered}
$$

Where $S_{j}^{*}$ and $S_{j}^{-}$are the positive and negative separation measures.

Step 4: The similarity index $\left(C_{j}^{*}\right)$ which is the similarity to the positive ideal solution for all the alternatives and the alternative with the highest $C_{j}^{*}$ value is selected as the best. $C_{j}^{*}$ is calculated as:

$$
C_{j}^{*}=\frac{S_{j}^{-}}{S_{j}^{*}+S_{j}^{-}} \quad 0 \leq C_{j}^{*} \leq 1
$$




\subsection{Assigning weights by pairwise comparisons}

With pairwise comparisons, criteria weights are determined by establishing the relative priority of each criterion against every other criterion in a matrix form [29]. The first row and column of the pairwise comparison matrix are filled with the criteria (i.e. each criterion appears in the first row and column of the matrix). The criteria in the columns are compared against the criteria in the rows and based on the judgment /preference of the decision maker, numerical ratings are assigned. The standard scale for assigning numerical ratings was developed by Thomas Saaty in 1980 and is shown in Table 2.

The upper triangular matrix is filled with numerical ratings from the scale, while the values in the lower triangular matrix are the inverse of the corresponding values in the upper triangular matrix. Next, the matrix is synthesized by summing up the values in each column, dividing each element by its column "total" and computing the average of the elements in each row. These average values are the "Priority Vector (PV)" or "Weights". To ensure consistency in the weightings, the Consistency Ratio (CR) is used. For CR $\leq$ 0.10 , the pairwise comparisons are relatively consistent and no corrective action is necessary; the reverse applies for $C R \geq 0.10$ and the sources of inconsistency must be identified and resolved [29, 30]. The CR is calculated as:

$$
\text { Consistency Ratio }(C R)=\frac{\text { Consistency Index }(C I)}{\text { Random Index }(R I)}
$$

Table 2: Saaty's scale of pairwise comparisons [30]

The Consistency Index measures the degree of inconsistency in the pairwise comparisons [31]:

$$
C I=\frac{\left(\lambda_{\max }-n\right)}{(n-1)}
$$

Where " $n$ " is the number of criteria and $\lambda_{\max }=\sum$ ( "column total" $x$ "corresponding PV" ) for all the criteria. The $\mathrm{Rl}$ is a direct function of the number of criteria or alternatives under evaluation and is obtained from a lookup table (Table 3) developed by Thomas Saaty in 1980 [29].

Table 3: Table of random indices [29] 


\section{Application of MCDA for Microalgae Strain Selection: Procedures and Assumptions}

\subsection{Procedure}

The core components of MCDA can be adapted to the microalgae strain selection problem. Fig. 3 shows the MCDA decision model for this MCDA problem. Six microalgae strains will be evaluated using six technical criteria (see table 1) in order to identify the best strain for biodiesel production. Table 4 shows a summary of the alternatives, criteria and input values used in this study; they are obtained from the work done by Anjorin [13] and Coward [15].

Fig. 3 Decision model for microalgae selection

\subsection{Assumptions}

The following assumptions are used in this study:

i. All the microalgae strains evaluated are grown under the same conditions in the same location.

ii. For simplicity, the cell size will be considered as the only determining factor for assigning the appropriate harvesting method for each microalgae strain. The cell sizes of the microalgae strains are presented in Table 5.

iii. Ease of harvesting is assumed to be directly proportional to the energy consumption rates of the harvesting methods. Therefore, lower energy costs indicate easier harvesting. The energy consumption rates are presented in Table 6.

Table 4: Criteria and alternatives

Table 5: Microalgae strains and their cell sizes [13]

Table 6: Energy consumption rates of harvesting methods [15] 


\section{Case Studies}

\subsection{Assigning weights}

Tables 7 and 8 show the pairwise and synthesized comparison matrices as described in section 3.3. From Tables 7 and $8, \lambda_{\max }=6.49$ and for $n=6$; applying equations (7) and (8), the $C R=0.079<0.1$. Therefore, the pairwise comparisons are consistent and the criteria weights can be used for further decision making. The weights of the criteria from table 8 are: Growth rate (0.29); Lipid content (0.40); PUFA (0.08); C16:1 (0.09); C18:1 (0.09); Ease of harvesting (0.04). Among the six criteria considered, the lipid content has the highest weight, followed by the growth rate. This implies that based on the decision maker's judgment (with data from literature etc.), the best strain of microalgae for biodiesel production has to have very high lipid content and a fast growth rate.

Table 7: Pairwise comparison matrix

Table 8: Synthesized (normalized) pairwise comparison matrix

\section{$5.2 \quad A H P$}

The AHP is performed in two steps:

Step 1: First, every alternative is assessed based on an individual criterion to obtain PVs. One pair-wise comparison matrix is constructed for every criterion and within each matrix, one alternative is compared to every other alternative [47]. Table 9 is the pairwise comparison matrix for "Growth rate". The matrices are normalized and the CR calculated to ensure consistency; Table 10 shows a collation of the PVs for the six criterion.

Step 2: For every alternative, the PVs from Table 10 are multiplied by the individual criterion weights. The sum of these products are the scores for each alternative. The alternative with the highest score is the best.

Table 9: Pairwise comparison matrix for "Growth rate"

Table 10: Priority vectors for the AHP

For ID001 the score is calculated as:

$(0.09 \times 0.29)+(0.08 \times 0.4)+(0.21 \times 0.08)+(0.43 \times 0.09)+(0.25 \times 0.09)+(0.17 \times 0.04)=0.14$

(c) 2015, Elsevier. Licensed under the Creative Commons Attribution-NonCommercial-NoDerivatives 4.0 International 
The scores for the other alternatives are:

ID002 $=0.28 ; \quad$ ID003 $=0.12 ; \quad$ ID004 $=0.1 ; \quad$ ID005 $=0.11 ; \quad$ ID006 $=0.25$

The best strain is ID002 (Scenedesmus sp.).

\section{$5.3 W S M$}

From Table 4, the positive criteria are growth rate, lipid content, $\mathrm{C} 16: 1$ and $\mathrm{C} 18: 1$; while the negative criteria are PUFA and energy consumption. First, the positive and negative criteria are transformed with equations 2 and 3. Finally, the rankings are calculated with equation 1 using the weights derived in section 5.1 . The weighted scores for the six microalgae strains are:

ID001 $=0.728 ; \quad$ ID002 $=0.796 ; \quad$ ID003 $=0.638 ; \quad$ ID004 $=0.632 ; \quad$ ID005 $=0.731 ; \quad$ ID006 $=0.725$

The best strain is ID002 (Scenedesmus sp.).

\section{$5.4 \quad W P M$}

Based on the six alternatives, the ideal strain of microalgae will have the following attributes:

$\mathrm{C} 1=0.492 \quad \mathrm{C} 2=30.09 \quad \mathrm{C} 3=4.91 \mathrm{C} 4=0.95 \quad \mathrm{C} 5=37.42 \quad \mathrm{C} 6=2.63$

Applying equation 4 to the data in Table 4, the scores are:

ID001 $=0.642 ; \quad$ ID002 $=0.582 ; \quad$ ID003 $=0.558 ; \quad$ ID004 $=0.669 ; \quad$ ID005 $=0.651 ; \quad$ ID006 $=0.489$

The best strain of microalgae from this method is strain ID004 (Chlorella vulgaris).

\section{$5.5 \quad D C P$}

Based on the six alternatives, the anti-ideal strain of microalgae will have the following attributes:

$\mathrm{C} 1=0.367 \quad \mathrm{C} 2=18.41 \quad \mathrm{C} 3=66.86 \mathrm{C} 4=0 \quad \mathrm{C} 5=0 \quad \mathrm{C} 6=7.57$

In this study, two scenarios will be considered in the DCP computation, " $p=1$ " and " $p=2$. Applying equations 5 and 6 to the data in table 4 , for $p=1$, the scores $\left(R_{i j}\right)$ are:

ID001 $=0.485 ; \quad$ ID002 $=0.223 ; \quad$ ID003 $=0.654 ; \quad$ ID004 $=0.718 ; \quad$ ID005 $=0.490 ; \quad$ ID006 $=0.293$ For $p=2$, the scores $\left(R_{i j}\right)$ are:

ID001 $=0.265 ; \quad$ ID002 $=0.122 ; \quad$ ID003 $=0.421 ; \quad$ ID004 $=0.400 ; \quad$ ID005 $=0.266 ; \quad$ ID006 $=0.147$

For $p=1$ and $p=2$, the best strain of microalgae is ID002 (Scenedesmus sp.). 


\subsection{TOPSIS}

The data in Table 4 are normalized and weighted by applying Equations 7 and 8, the results are shown in Tables 11 and 12. The positive and negative ideal solutions are:

$$
\begin{array}{ll}
A^{*}=\left\{v_{1}^{*}, v_{2}^{*}, \ldots, v_{i}^{*}, \ldots, v_{n}^{*}\right\} & =\{0.492,30.09,4.91,0.95,37.42,2.63\} \\
A^{-}=\left\{v_{1}^{-}, v_{2}^{-}, \ldots, v_{i}^{-}, \ldots, v_{n}^{-}\right\} & =\{0.367,18.41,66.86,0,0,7.57\}
\end{array}
$$

Applying equations 9,10 and 11 , the $C_{j}^{*}$ values for the alternatives are:

$\mathrm{ID} 001=0.590982 ; \quad \mathrm{ID} 002=0.590976 ; \quad \mathrm{ID} 003=0.590876 ;$

ID004 $=0.591208 ; \quad$ ID005 $=0.591041 ; \quad$ ID006 $=0.590928$

The best microalgae strain is strain ID004 (Chlorella vulgaris).

Table 11: Normalized criteria $\left(\bar{r}_{i j}\right)$

Table 12: Weighted normalized criteria $\left(v_{i j}\right)$

\subsection{Discussions}

Table 13 is a summary of the results from the five MCDA methods used in this study for microalgae strain selection. Four out of the six results agree that strain ID002 is the best strain and all six results agree that the $3^{\text {rd }}$ best strain is strain ID001. The results are however not uniform for the second best strain. It is also worthy of note that no two methods have produced identical ratings.

From the results, it is observed that ID002 has the best properties with respect to the lipid content and energy consumption. ID002 also has a fast growth rate compared to other strains. ID005 and ID006 (both second place) also have very high lipid contents, their energy consumption rates are the second best and the growth rate of ID006 is very high. ID001 has the $3^{\text {rd }}$ highest lipid content and a low energy consumption rate, the growth rate is also comparatively high.

From literature, it is noted that the strains with higher lipid contents and faster growth rates are good feedstock for biodiesel production. Comparing these results with the observations from literature, the strains with the higher lipid contents and growth rates ranked very highly in the results. The strains that were easier 
to harvest (based on energy consumption) also ranked highly - ID002 has the least energy consumption value of all the strains.

Table 13: Summary of results

\section{Conclusions and Recommendations for Future Work}

The selection of the best strain of microalgae is an important step in the microalgae-to-biodiesel production process, as this has a direct effect on the yield and properties of the biodiesel produced. Five MCDA methods have been used to select the best strain from six microalgae strains. The criteria used were growth rate, lipid content, fatty acid profile and ease of harvesting. The strain with the best properties as decided by three of the five methods is Scenedesmus $s p$.

From this study, the lipid content is the most important factor to be considered in the selection of the best microalgae strain for biodiesel production because it has the highest relative importance factor (weight) among all the criteria. According to the results, the best strain has the highest lipid content; the second and third best strains also have the second and third highest lipid contents respectively. Therefore, increasing the lipid contents of microalgae strains should be a major research area for microalgae-based research.

The growth rate is also a very important criterion in the selection of microalgae strains. The strains with comparatively high growth rates ranked among the first to third best. Energy consumption during harvesting also plays a very important role in the strain selection as the strains with the lowest energy consumption rates are ranked best among the alternatives.

It is unclear from the results, how the fatty acid profile affects the strain selection. The relationship between the weights and input values of the PUFA, C16:1 and C18:1 do not follow any observed patterns. The fatty acid profile however has a very significant effect on the physical and chemical properties of the biodiesel produced and hence should be a very important part of the selection process.

In light of the above conclusions, the following aspects are recommended for future work:

- More criteria should be added to the decision models to expand the scope of the decision making process.

(C) 2015, Elsevier. Licensed under the Creative Commons Attribution-NonCommercial-NoDerivatives 4.0 International 
- In order to draw robust conclusions from the results, sensitivity analysis should be carried out. The sensitivity analysis will determine how variations in the criteria weights and inputs will influence the final scores and hence the final decisions.

- The strengths and the weaknesses of the MCDA methods used in this study should be investigated. The characteristics of the MCDA methods may have a significant impact on the rankings of the alternatives.

- The role of the fatty acid profile in microalgae strain selection should be further studied. Some work has been done on this subject [12, 32] but different approaches can be considered.

- An evaluation tool can be developed using the MCDA methods analysed in this study to make the microalgae selection process easier and enable the comparison of a large number of strains simultaneously.

\section{References}

[1] Nwokoagbara E. 2011. Technical and economic analysis of biofuel production processes from microalgae, MSc. Thesis. Cranfield University, Cranfield, United Kingdom

[2] Wang B., Li Y., Wu N., Lan Q. $\mathrm{CO}_{2}$ bio-mitigation using microalgae. Applied Biotechnology 2008; 79:707-718

[3] Cobuloglu H., Guan Y., Liao H. A multi-criteria approach for biomass crop selection under fuzzy environment. Proceedings of the 2014 Industrial and Systems Engineering Research Conference. Kansas, USA; 2014

[4] Viswanath B., Mutanda T., White S., Bux F. The Microalgae - A future source of biodiesel. Dynamic Biochemistry, Process Biotechnology and Molecular Biology 2010; 4(1): 37 - 47

[5] Mata T.M.; Martins A.A.; and Caetano N.S., Microalgae for biodiesel production and other applications: A review. Renewable and Sustainable Energy Reviews (2010) 14: 217 - 232

[6] Sun X., Li Y. An intelligent multi-criteria decision support systems design. American institute of aeronautics and astronautics. pp. 1-11 
[7] Linkov I., Steevens J. Chapter 35 Appendix A: multi-criteria analysis. ADVANCES IN EXPERIMENTAL MEDICINE AND BIOLOGY; Cyanobacterial harmful algal blooms; state of the science and research needs Symposium. (2008) 619: 815 - 830

[8] Department for Communities and Local government: London. Multi-criteria analysis: a manual. Crown copyright 2009. ISBN: 978-1-4098-1023-0

[9] Mohindru, P. (2011). Fuzzy multi-criteria based decision making problems in Indian IT industry: a comparative study of TCS and HCL. Retrieved from Indian ETD Repository. Available at: http://shodhganga.inflibnet.ac.in/handle/10603/2896 Accessed July 2014

[10] Griffiths M., Harrison S. Lipid productivity as a key characteristic for choosing algal species for biodiesel production. Journal of Applied Phycology 2009; 21(5): 493-507

[11] Oilgae. Algal oil yields. Available at: http://www.oilgae.com/algae/oil/yield/yield.html Accessed May 2014

[12] Iracema A.N., Marques S.S., Cabanelas I.T., Pereira S.A., Druzian J.I., Oliveira de Souza C., Vich D.V., Correia de Carvaalho G., Nascimento M.A. Screening microalgae strains for biodiesel production: Lipid productivity and estimation of fuel quality based on fatty acid profiles as selective criteria. Bioenergy Research 2013; 6:1-13

[13] Anjorin R. (2011) Selection of microalgae strains for biodiesel production. MSc. Thesis. Cranfield University, Cranfield, United Kingdom

[14] Stansell G., Gray M., Sym D. (2011) Microalgal fatty acid composition: Implications for biodiesel quality. Journal of Applied Phycology. Available at:

http://www.springerlink.com/content/?k=Microalgal+fatty+acid+composition\%3a+implications+for+ biodiesel+quality Accessed June 2014

[15] Coward T., Lee J.G.M., Caldwell G. S. Development of a foam flotation system for harvesting microalgae biomass. Algal Research 2013; 2: 135 - 144

[16] Molina Grima E., Belarbi E., Acién F. G., Robles Medina A., Chisti Y. Recovery of microalgal biomass and metabolites: Process options and economics. Biotechnology Advances 2003; 20 (78): $491-515$ 
[17] Maharajh D., Harilal A. (2010), Transforming South Africa's biodiversity into diesel. Available at: http://researchspace.csir.co.za/dspace/bitstream/10204/4234/1/Maharajh 2010.pdf Accessed June 2014

[18] Singh A., Olsen I. A critical review of biochemical conversion, sustainability and life cycle assessment of algal biofuels. Applied Energy 2011; 88 (10): 3548-3555.

[19] Bazaes J., Sepulveda C., Gabriel Acien F., Morales J., Gonzales L., Rivas M., Riquelme C. Outdoor pilot-scale production of Botryococcus braunii in panel reactors. Journal of Applied Phycology 2012; DOI 10.1007/s10811-012-9787-3

[20] Keely J.W., (2005) Culvert Pipe Liner Guide and Specifications: Chapter 5 - Multi-criteria decision analysis software. Publication No. FHWA - CFL/TD - 05 - 003. pp. 87 - 101

[21] Triantaphyllou E., Mann S. H. An examination of the effectiveness of multi-dimensional decisionmaking methods: a decision-making paradox. Decision Support Systems 1989; 5: $303-312$

[22] Pohekar S.D., Ramachandran M. Application of multi-criteria decision making to sustainable energy planning - A review. Renewable and Sustainable Energy Reviews 2004; 8: 365 - 381

[23] Topcu Y.I. (2004) Chapter 3 'SAW, WP, TOPSIS' [PowerPoint slides]. Available at: http://web.itu.edu.tr/topcuil/ya/END332E MADM.pptx Accessed July 2014

[24] Podvezko V. The comparative analysis of MCDA methods of SAW and COPRAS. Engineering Economics 2011; 22(22): 134-146

[25] Ishizaka A., Nemery P. (2013) Multi-criteria decision analysis: methods and software. Wiley, UK

[26] Mohindru, P. (2011). Fuzzy multi-criteria based decision making problems in Indian IT industry: a comparative study of TCS and HCL. Retrieved from Indian ETD Repository. Available at: http://shodhganga.inflibnet.ac.in/handle/10603/2896 Accessed July 2014

[27] Azar F.S. Multiattribute decision-making: use of three scoring methods to compare the performance of imaging techniques for breast cancer detection (2000). Technical Reports (CIS). Paper 119. Available at: $\underline{\text { http://repository.upenn.edu/cis reports/119 }}$

[28] Maldonado R.E., Delabastita W., Wijfels A., Orshoven J.V. Comparison of discrete multi-criteria decision making methods for selection of afforestation sites. SAGEO 2012; pp. 1 - 16 
[29] Kunz J. The analytic hierarchy process (AHP). Eagle City Hall Location Options Task Force. February/March 2010

[30] Murphy S. (2005) Analytical Heirarchy Process - Part 1. [PowerPoint slides]. Available at: http://www.udel.edu/UMS/itv/2001S/buad346/ Accessed June 2014

[31] Sarfaraz A.R., (2006) Multicriteria Decision Making: Analytical Heirarchy Processes. [PowerPoint slides] Available at: http://www.ecs.csun.edu/ sarfaraz/606B-wk4.ppt Accessed March 2014

[32] Islam M.A., Magnusson M., Brown R.J., Ayoko G.A., Nabi M.N., Heimann K. Microalgal species selection for biodiesel production based on fuel properties derived from fatty acid profiles. Energies 2013; 6: 5676-5702 